\title{
Design and Simulation Analysis of Mechanical Picking Comb Teeth of Artificial Finger Marigold Flowers
}

\author{
Qi Zhengdong ${ }^{1}$, Li Liangliang ${ }^{*}{ }^{*}$, Lian Guodang $^{1}$, Li Ping $^{1}$, Liu Xiangdong $^{1}$ \\ ${ }^{1}$ Xinjiang Institute of Technology,School of Electrical and Mechanical Engineering,843100 Xinjiang Aksu, China
}

\begin{abstract}
As the key component of mechanical picking device of marigold flower, the structure, size, strength and motion of comb are closely related to the reliability and the rate of picking. In this paper, a mechanical comb for marigold based on bionic finger is designed, and the parameters of structure, row, spacing and outside diameter are designed respectively. Then, the three-dimensional model is established with ANSYS/LS-dyna platform, and the design strength of the comb-type picking mechanism is checked. The results show that the maximum deformation of the comb with $2.3802 \mathrm{~mm}$ occurs near the top of the tooth during the picking process, which is small and meets the design strength requirements. Finally, the picking process of the comb-type picking mechanism is simulated and the result shows that when the operating speed $\mathrm{v}$ is set to $3.0 \mathrm{~km} / \mathrm{h}$ and $\mathrm{w}$ is set to $30 \mathrm{r} / \mathrm{min}$, the picking effect is good. The above research provides a theoretical reference for the mechanical picking of marigold.
\end{abstract}

\section{Introduction}

Marigold is an important flower widely used in industry and medicine for its economic value. Especially, the pigments in marigold have obvious medicinal benefits on anti-cancer, anti-aging, anti-human immunodeficiency virus, so the attention and demand for marigold flower is increasing, attention is increasing with the result that marigold is planted in large quantities $^{[1,2]}$.At present, marigold mainly relies on manual picking, which costs huge labor with low efficiency, high cost is, time-consuming and pollen allergy, especially susceptible to weather factors. Apart from that, marigold should be treated with anti-oxidation and preservation immediately after picking, which could cause huge economic loss with incorrect operation ${ }^{[3,4]}$. Presently, the research on mechanical picking of marigold mainly focuses on small-scale hand-held or backpack picking mechanism with low level of mechanization, which is not applicable to the large-scale operation. The mechanism does not improve the picking efficiency fundamentally, but increases the workload to a certain extent. Consequently, a mechanical comb for marigold based on bionic finger is designed, and the mechanical properties of the mechanism are analyzed and the picking process is simulated based on ANSYS/LS-dyna platform.

\section{Structure design of comb}

\subsection{Structure design}

The hand-picking of marigold is the most labor-intensive work, of which the flowers are mostly of the best quality. It is best to pinch the pedicel under the petal by finger, and the pedicel should not exceed $1 \mathrm{~cm}^{[5,6]}$. The design of comb structure chooses the bionic finger and the anterior joint of fingertip is bent for fixed radian, which is more advantageous to the plant carding and collecting after picking

\subsection{Design of comb row}

The planting density of marigold is about 33,000-36,000 plants $/ \mathrm{hm} 2$ with the row spacing about $500 \mathrm{~mm}$, the plant spacing about $38 \mathrm{~cm}$, the number of branches (branch) about 15, and the number of effective flowers (flowers) per plant about 70. Because the chrysanthemum is denser on the single plant, considering the tractive speed of tractor in the field, the operating speed of the picking mechanism is preset to $0.3 \mathrm{~m} / \mathrm{s}$, and the rotating speed of picking comb is $30 \mathrm{r} / \mathrm{min}$.

It can be seen from the formula (1) that the rotation speed is inversely proportional to the rows of comb. Therefore, the greater the rotating speed is, the fewer rows of picking comb should be set. However, if the speed is too high, entangling and idling is more likely to happen, so the speed should not be too large. Besides, if the rotating speed is fixed, the more the rows of comb is, the interval of comb picking time is shorter ${ }^{[7]}$. Formula (1) is required to ensure that each flower will not be missed when picking between the individuals.

\footnotetext{
* Corresponding author: Li Liangliang (1990-), male, lecturer, Master of Engineering, majoring in agricultural mechanization research. E-mail: 1110721vip@163.com.
} 


$$
t \leq \frac{l}{v}=\frac{0.35 m}{0.5 m / s}=0.7 \mathrm{~s}
$$

where in formula (1), $l$ denotes the-plant spacing, $\mathrm{mm} ; v$ denotes the machine operating speed.

In order to ensure that marigold chrysanthemum can be picked every time, the interval of comb and the rotating speed and the rows of comb should be satisfied the formula (2):

$$
t=\frac{60}{k \cdot n}
$$

where in formula (2), $t$ denotes the interval, $\mathrm{s} ; k$ denotes the rows of comb; $n$ denotes the rotating speed, $r / \min$.

$$
0.7 s=\frac{60}{k \cdot 40 r / \min }, \text { result } k \text { is } 2.1 .
$$

Considering the reasonable rotational speed of the rotating shaft and the structure dimensions, the equation (1) condition is satisfied with $t$ of $0.67 \mathrm{~s}$ when $k$ is 3 . However, $t$ is close to 0.7 , which is not the most appropriate value, so setting $k$ to 4 .

\subsection{Design of comb spacing}

When picking the flower, the picking comb gathers the branches of marigold and feed them into the separating teeth, and the branches beyond the end face are drawn into the gap between the comb teeth. And, if the gap between the teeth is too large, the flowers will be easily missed. While too small gap can cause branch entangling, misoperation, clogging and great resistance.

Taking the symmetrical force of single flower feeding as an example, the mechanical analysis of the comb in the picking process of marigold is carried out. To ensure that the flowers are picked off, the formula $2 T_{y} \geq R$ must be satisfied. In the formula, $T_{y}$ is the component of the impact force of the comb rod on the flower in the $\mathrm{Y}$-axis direction, which is calculated as follows: (3) ${ }^{[8]}$, N. $R$ is the flower picking force, N.

$$
T_{y}=T \cdot \sin \alpha=T \cdot \frac{h}{\sqrt{h^{2}+\left(\frac{d}{2}\right)^{2}}}
$$

The tensile test shows that the flower was stretched between the picking comb and the separating teeth in the picking process, and it was pulled off at the pedicel. And, the picking comb and the receptacle are also impacted. Combined with the measured value of stem diameter of marigold straw of $7 \sim 10 \mathrm{~mm}$ and the diameter of the main flower of 8 9.5 mm, the comb gap is larger than the stem diameter and smaller than the receptacle diameter, so the theoretical range value of $d$ is: $9 \leq d \leq 11$ and the design value is set to $10 \mathrm{~mm}$. Considering the problems of entangling and clogging in the process of picking, the optimal gap of picking comb teeth should be determined by experiments.

\subsection{Design of the comb outside diameter}

The picking comb is mounted on the comb mounting base of the rotating shaft, following the rotating shaft and the frame. The pick comb motion is the composite motion of the rotation of the rotating shaft and the forward motion of the frame. In order to ensure that both the highest and lowest flowers on the individuals can be picked, the outside diameter $R$ of the picking comb should satisfy the formula (4). Moreover, during the rotation of the rotating shaft ${ }^{[9]}$.

$$
R \geq\left|\frac{y-h}{\sin \omega t}\right|
$$

where, $R$ denotes the comb outside diameter, $\mathrm{mm}, h$ denotes the-installation height of rotating shaft, $\mathrm{mm}, y$ denotes the-plant average height, $\mathrm{mm}, \omega$ denotes the angular velocity, $\mathrm{rad} / \mathrm{s}$.

The radius of picking comb should be greater than the height difference of flowers to ensure that all the chrysanthemum can be picked without omission. $|y-h|$, the distance between the plant height and the comb shaft, should be greater than the extreme difference of the height. The mean of flower height difference in the same horizontal area is $70 \mathrm{~mm}$ by measurement, and the design value of the comb outside diameter is set to $83 \mathrm{~mm}$.

According to the above analysis, the structure of the comb mechanism is shown in Figure 1. The bionic finger comb teeth are arranged to form a finger rack. Each root of the teeth is provided with a cutter. The left and right sides of the outside wall of the cutter are respectively equipped with a knife blade. The V-shape cutting channel is formed between the teeth, which is broad at front part and narrow at back part. And the comb teeth are inserted into the inside of the marigold for carding. Being acted by the combing force, the flowers fall off along the gap between the comb teeth to the root of the teeth and enter the cutting channel eventually. Then, the joint of the flower and the stem is cut by the blade on the root of the teeth. The comb structure is finally installed in the cylinder picking mechanism for picking.

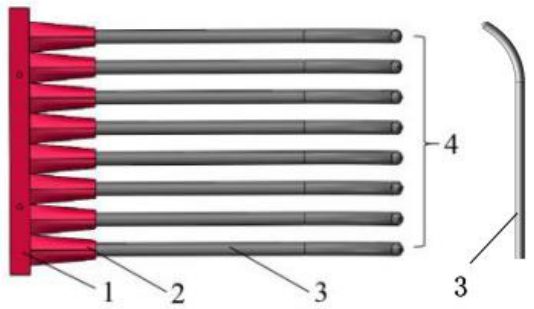

1. Finger rack 2. V-shaped cutter 3. Bionic finger comb 4. Finger row

Fig. 1. 3-D view of comb-type picking mechanism

\section{Simulation analysis of mechanics and kinematics}

\subsection{Analysis of mechanical properties of comb}

As the key component of the picking device, Too many flowers result in the increase of the acting force on the single tooth in the picking process, and the comb is prone to deflection and deformation. Accordingly, it is necessary to simulate and analyze the stress and strain of 
the comb in the process of picking, and evaluate the strength of the mechanism according to the results.

The three-dimensional model of comb teeth built in SolidWorks is imported into the finite element analysis software ANSYS for mechanical analysis, and the finite element model of solid element is established using the three-dimensional four-dimensional block element with the size of $10 \mathrm{~mm}$, which is selected to simulate the curved-surface solid on the boundary to improve the accuracy of the simulation calculation. Then, the model is meshed by free meshing on a granular level, and the method of local mesh refined is applied on the local important parts with stress distribution, such as the through-hole of the toolholder. The comb material is set to $\mathrm{Cr} 12 \mathrm{MoV}$, of which the density is $7.85 \mathrm{~g} / \mathrm{cm}^{3}$, Poisson's ratio is 0.25 and Young's modulus is $2.5 \times 10^{5} \mathrm{Mp}$. Besides, the comb is fixed through the through-hole of the tool-holder, and the fixed constraint is added to the tool-holder. During the simulation, a load perpendicular to the tangent direction of the elbow is applied at the elbow of the front end of the comb to check the strength of the mechanism, and the mechanical simulation result is as shown Fig. 2 .

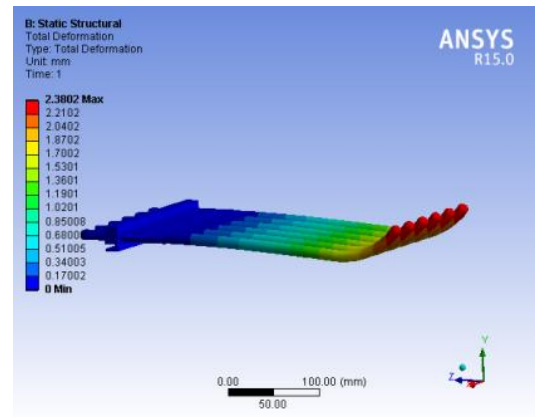

Fig. 2. Diagram of comb deformation

It can be seen from the diagram of comb deformation that the deformation near the through-hole of the toolholder is small, the displacement from the root to the top of the teeth increases gradually, and the maximum displacement is $2.3802 \mathrm{~mm}$ near the top part. It can be concluded that the maximum deformation of the comb appears near the top of the teeth in the picking process and the value is small, which meets the strength requirements of the mechanism.

\subsection{Simulation analysis of the picking process}

Based on ANSYS/LS-dyna platform, the picking process of the comb-type picking mechanism is simulated and analyzed. And, the following hypotheses on plant characteristics are made: neglecting the bending and sagging, supposing the branches and flower as erect state, ignoring the uneven diameter of straw, setting the plant as isotropic flexible body, neglecting the collision and deformation between flower and comb in the picking process, neglecting the influence of the external factors such as flowers, leaves and branches on the motion. Besides, the setting of the rigid comb material properties is the same as that of the mechanical property material. And, the density of the flexible flower is $400 \mathrm{~kg} / \mathrm{m}^{3}$, Poisson's ratio is 0.3 , the elastic modulus is $90 \mathrm{MPa}$, and the maximum picking force is $5.5 \mathrm{~N}$. The horizontal linear velocity v of comb is as follows: $1.5 \mathrm{~km} / \mathrm{h}, 3.0 \mathrm{~km} / \mathrm{h}$, $4.5 \mathrm{~km} / \mathrm{h}$, and the rotating angular velocity is as follows:30r/min, $40 \mathrm{r} / \mathrm{min}, 50 \mathrm{r} / \mathrm{min}$. The simulation of the picking process with the relevant parameters is shown in Figure 2.
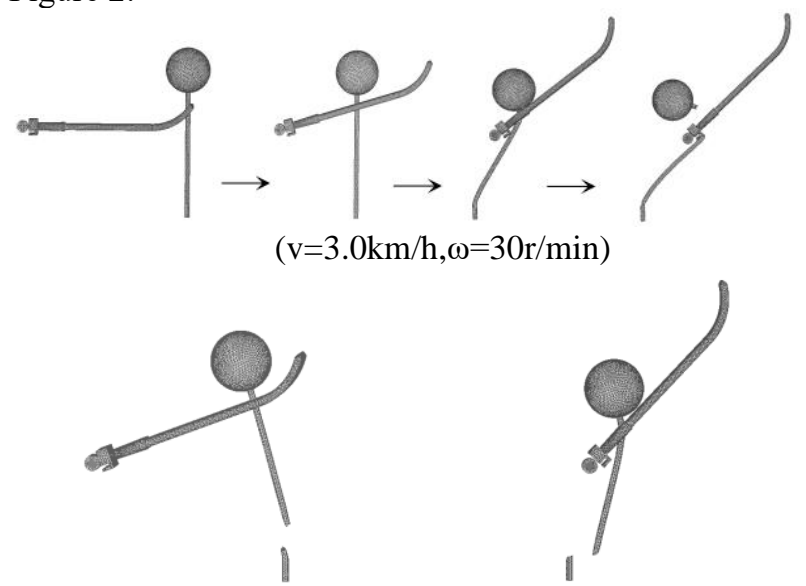

$(\mathrm{v}=1.5 \mathrm{~km} / \mathrm{h}, \omega=50 \mathrm{r} / \mathrm{min}) \quad(\mathrm{v}=4.5 \mathrm{~km} / \mathrm{h}, \omega=40 \mathrm{r} / \mathrm{min})$

Fig. 3. Simulation of the comb picking process

As the simulation experiment shows, after the marigold plants contact with the comb, the plants mainly go through the combing, tearing and cutting processes under the action of comb. When $v$ is $3.0 \mathrm{~km} / \mathrm{h}$ and $\omega$ is $30 \mathrm{r} / \mathrm{min}$, the picking effect is good, which can realize the ideal picking. But when $v$ is $1.5 \mathrm{~km} / \mathrm{h}$ and $\omega$ is $50 \mathrm{r} / \mathrm{min}$, the angular velocity is big and, linear velocity is small, resulting in great centripetal pulling force. The comb pulls the marigold plant to the left with the force and fail to pick the flower. Moreover, when $v$ is $4.5 \mathrm{~km} / \mathrm{h}$ and $\mathrm{w}$ is 40r/min, the linear velocity is big and the angular velocity is small, resulting in great horizontal pulling force, so the comb pull the marigold plant to the right and also fail to pick the flower. Therefore, from the simulation analysis, the setting of $\nu=3.0 \mathrm{~km} / \mathrm{h}$ and $\omega=30 \mathrm{r} / \mathrm{min}$ is more reasonable. And, if the design value is lower than that, the picking can only be finished with low efficiency, so the specific value needs to be determined by further field experiments.

\section{Summary}

A comb-type picking mechanism of marigold flower based on bionic finger is proposed, in which the rows of comb is 4 , the clearance is $10 \mathrm{~mm}$ and the outside diameter of comb was $83 \mathrm{~mm}$. Based on ANSYS/LSdyna platform, the design strength of the comb-type picking mechanism is checked. The results show that the maximum deformation of $2.3802 \mathrm{~mm}$ occurs near the top of the teeth in the picking process, and the value is relatively small which meets the strength requirements of the mechanism. Moreover, the virtual picking process of the comb-type picking mechanism is carried put. And, the results show that the speed setting of $\nu=3.0 \mathrm{~km} / \mathrm{h}, \omega=30 \mathrm{r} / \mathrm{min}$ is reasonable and the picking effect is good. The conclusion provides a theoretical reference for the mechanical picking of marigold. 
This article is one of the phased results of the 2020

Xinjiang Uygur Autonomous Region University Scientific Research Project Fund Youth Project 《A Study on the Mechanism and Method of Comb Marigold Mechanized Picking Based on ADAMS/ANSYS/LSDYNA》(Project No.: XJEDU2020Y048)

\section{References}

1. Tan Meiwei, Li Guoyu, Lv Xinyu, et al. Review of tagetes erecta L. on the components and pharmacological action $[\mathrm{J}]$. Information on Traditional Chinese Medicine, 2017, 34(06): 138141.

2. Zhao Dezhu, Li Yunhai, Chen Lihua, et al. Application value and high-yield cultivation techniques of marigold[J]. Modern Agricultural Science and Technology, 2010, (13):146-150.

3. Niu Guoling, Jiang Yongcheng, Li Biaobiao, et al. Status and analysis of mechanical marigold picking [J]. Mechanical Engineer, 2014(05): 47-49.

4. Niu Guoling, Jiang Yongcheng, Li Biaobiao, et al. Analysis of the current situation of mechanical picking of marigold[J]. Mechanical Engineer, 2014, (5): 32-34.

5. Cai Chunmei. A brief discussion on the cultivation and management technology of marigold [J]. Nong Min Zhi Fu Zhi You, 2017(06): 157-158.

6. Zhang Xinmin. Management and picking of marigold in the middle and late stages[J]. Rural Science and Technology, 2016, (11):53-53.

7. Ji Changying, Zhang Chun, Gu Baoxing, Fu Haijiao, Xie Di, Guo Jun, et al. Design and experiment of shear-sucting mountain chrysanthemum picking machine $[\mathrm{J}]$. Transactions of the Chinese Society for Agricultural Machinery, 2017, 48(11): 137-145.

8. Ji Changying, Wang Chunxiang, Gu Baoxing, Zhang Chun, et al. Design and experiment of handpush chrysanthemum morifolium comb-picking machine $[\mathrm{J}]$. Transactions of the Chinese Society for Agricultural Machinery, 2016, 47(07): 143-150+142.

9. Cao Weibin, Sun Weiling, Niu Chi, et al. Combed safflower picking device based on ANSYS/LSDYNA [J]. Transactions of the Chinese Society for Agricultural Machinery, 2018, 49(11): 123-131. 